UCRL-ID-121109

\title{
Derivation of Plutonium-239
}

Materials Disposition Categories

\author{
W. G. Brough
}

April 27, 1995
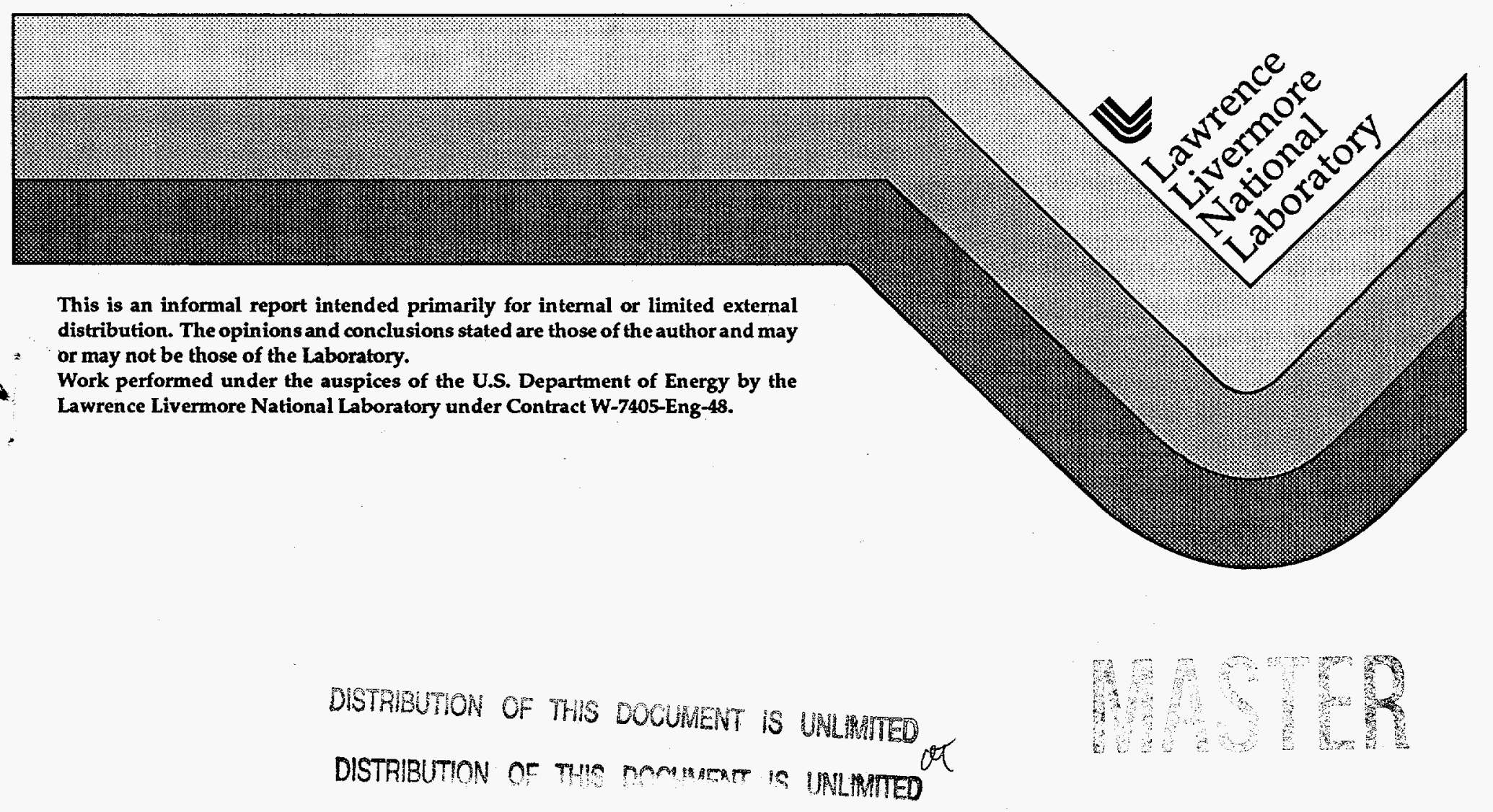


\section{DISCLAIMER}

This document was prepared as an acoount of work sponsored by an agency of the United States Government. Neither the United States Government nor the University of California nor any of their employees, makes any warranty, express or implied, or assumes any legal liability or responsibility for the accuracy, completeness, or usefulness of any information, apparatus, product, or process disclosed, or represents that its use would not infringe privately owned rights. Reference herein to any specific commercial product, process, or service by trade name, trademark, manufacturer, or otherwise, does not necessarily constitute or imply its endorsement, recommendation, or favoring by the United States Covernment or the University of California. The views and opinions of authors expressed herein do not necessarily state or reflect those of the United States Government or the University of California, and shall not be used for advertising or product endorsement purposes.

This report has been reproduced directly from the best available copy.

Available to DOE and DOE contractors from the Office of Scientific and Technical Information P.O. Box 62, Oak Ridge, TN 37831

Prices available from (615) 576-8401, FTS 626-8401

Available to the public from the

National Technical Information Service

U.S. Department of Commerce

5285 Port Royal Rd.

Springfield, VA 22161

Work performed under the auspices of the U.S. Department of Energy by Lawrence Livermore National Laboratory under Contract W-7405-ENG-48. 


\section{DISCLAIMER}

Portions of this document may be illegible in electronic image products. Images are produced from the best available original document. 


\section{Derivation of Plutonium-239 Materials Disposition Categories}

April 27, 1995

Winslow G. Brough

Lawrence Livermore National Laboratory

NAI/Systems Modeling and Analysis 


\begin{abstract}
At this time, the Office of Fissile Materials Disposition within the DOE, is assessing alternatives for the disposition of excess fissile materials. To facilitate the assessment, the Plutonium-Bearing Materials Feed Report for the DOE Fissile Materials Disposition Program Alternatives $(U)^{1}$ report was written. The development of the material categories and the derivation of the inventory quantities associated with those categories is documented in this report.
\end{abstract}

\title{
Pre-Existing Material Categories
}

The DOE inventory of plutonium exists in a number of different forms (i.e., metals, alloys, and compounds), and in varying degrees of isotopic enrichment and chemical purity. The material form, enrichment, and types and levels of impurities are considered when deciding the appropriate disposition of any excess plutonium. Material categories were devised to facilitate the characterization of processing requirements for dispositioning material through any given disposition alternative.

The basic inventory data is available at the site level, and at the DOE-wide level within the Office of Arms Control and Nonproliferation, which maintains the Nuclear Materials Management and Safeguards System (NMMSS). The individual site databases provide detailed information on each physical inventory item, but the categorization schemes are not standardized or consistent from site to site. The NMMSS provides less detailed information on materials, but is, instead, summarized by categories and is more consistent in its scheme of categorization. Given the inherent difficulty in correlating and assessing information from the site databases, and the desire to maintain consistency with the DOE-wide plutonium inventory database, the NMMSS database was used as the basis for the feed report. The NMMSS has a categorization scheme that identifies ranges of isotopic enrichment, the general makeup of the plutonium inventory based on the most recent processing activity, and the program for which the material is being provided.

Seven isotopic assay categories are used in the NMMSS database to.identify the isotopic assay of ${ }^{240} \mathrm{Pu}$ in the Plutonium-239 material. This characteristic is used to differentiate weapons-grade . plutonium from reactor-grade plutonium. The Isotopic Range Codes, or Material Types, 51 and 52 are considered weapons-grade. The assay ranges for the isotopic assay categories are given in the following table:

\begin{tabular}{|c|l|}
\hline Isotopic Range Code & \multicolumn{1}{|c|}{ Range $\left(\%{ }^{240} \mathrm{Pu}\right)$} \\
\hline 51 & $<4.00$ \\
\hline 52 & 4.00 to $<7.00$ \\
\hline 53 & 7.00 to $<10.00$ \\
\hline 54 & 10.00 to $<13.00$ \\
\hline 55 & 13.00 to $<16.00$ \\
\hline 56 & 16.00 to $<20.00$ \\
\hline 57 & 20.00 and above \\
\hline
\end{tabular}

Table 1. Isotopic Assay Range Codes for Plutonium-239.

The general makeup of plutonium in the NMMSS database is cataloged into one of approximately 40 main categories and 200 sub categories known as Composition of Ending Inventory (COEI) codes.

\footnotetext{
${ }^{1}$ UCRL-ID-120749, Winslow G. Brough (LLNL), Stephen T. Boerigter (LANL), April 6, 1995
} 
Additionally, the seven sub categories for Unirradiated Scrap Material Awaiting Recovery ( COEIs 721-722, and 725-729), are further subdivided into another approximately 130 sub categories, called ANSI scrap codes (referring to the American National Standards Institute ANSI N15.10-1987).

For the most part, the NMMSS COEI codes and descriptors did not sufficiently characterize the plutonium inventory to easily identify processing requirements for the purposes of material disposition. The.ANSI scrap codes provided more detailed descriptions for those materials identified as scrap, but the NMMSS database was still lacking information pertaining to the levels of alloying constituents and impurities. Other sources of information from the sites and from DP-22 were used to gain a better understanding of typical alloying constituents and impurities.

In a search for a Materials Disposition categorization scheme, individual site item description codes (e.g., RFP's IDC, LANL's IDES, and LLNL's forms and shapes) were reviewed and found inconsistent for a DOE-wide assessment and roll-up. It was decided to start with a categorization scheme used by the DOE Defense Programs (DP-22) that used nine main categories. Specific COEI, ANSI, and project codes had been assigned to the nine main categories as shown in Table 2. Not only were the DP-22 categories close to the needs of Materials Disposition, but by using the DP-22 categories, it helped in separating those materials that.were excess to Defense Programs from those that were not. 


\begin{tabular}{|c|c|}
\hline DP-22 Category & COEI and ANSI codes included in category \\
\hline Pits & 309 (-GB- only), $580,581,582,699,772$ \\
\hline $\begin{array}{l}\text { Metal -- } \\
\text { Unalloyed }\end{array}$ & $\begin{array}{l}132,134,145,147,148,150,152,154,164^{\circ}, 165,167,169,180,182,184, \\
195,197,200,204,206,220,222,224,236,237,238,251,252,254,305 \\
(-G B-), 308 \text { (-GB-), 450, 690, 701, 721 (A00, A02, \& A03), 778, 779, 782 }\end{array}$ \\
\hline Metal -- Alloyed & $\begin{array}{l}133,146,149,151,153,155,166,168,170,181,183,185,196,198,201 \\
205,207,221,223,225,253,255,256,257,306 \text { (-GB-), } 691,702,722 \\
(B 00, \mathrm{~B} 02, \mathrm{~B} 03, \mathrm{~B} 11, \mathrm{~B} 40, \mathrm{~B} 70, \& \mathrm{~B} 90), 780,783\end{array}$ \\
\hline Oxides & $\begin{array}{l}060,061,062,235,287,289,453,454,455,456,457,635,636,637,709 \\
710^{*}, 725(\mathrm{C} 00, \mathrm{C} 01, \mathrm{C} 02, \mathrm{C} 41, \mathrm{C} 71, \& \mathrm{C} 90)\end{array}$ \\
\hline $\begin{array}{l}\text { Other } \\
\text { Compounds }\end{array}$ & $063,064,081,082,083,130,131,410,572,692,770,775,776,784$ \\
\hline $\begin{array}{l}\text { Nitrate } \\
\text { Solutions }\end{array}$ & $409,452,703$ \\
\hline $\begin{array}{l}\text { R\&D, Reactor } \\
\text { Fuel, and } \\
\text { Miscellaneous }\end{array}$ & $\begin{array}{l}285,286^{*}, 288,290,291,305 \text { (non-GB-), } 306 \text { (non-GB-), } 307 \text { (non-GB-), } \\
309 \text { (non-GB-), 481, 745, 746, 747, 748, 749, 752, 771, 774, 781, 785, 788, } \\
789,836\end{array}$ \\
\hline Scrap / Residues & $\begin{array}{l}693,694,695,696,697,700,721 \text { (except A00, A02, \& A03), 722 (except } \\
\text { B00, B02, B03, B11, B40, B70, \& B90), 725 (except C00, C01, C02, C41, } \\
\text { C71, \& C90), 726, 727, 728, 729, 777, } 791\end{array}$ \\
\hline Irradiated $\mathbf{F}$ & $360,362,363,375,376,385,386,387,389,390,391,392,405,408,790$ \\
\hline
\end{tabular}

Table 2. DP-22 Plutonium Composition Categories by $\mathrm{COEI}^{2}$ and $\mathrm{ANSI}^{3}$ Codes

DOE Material Disposition Categories

The nine DOE/DP-22 categories were expanded to eleven categories for the DOE/MD categorization scheme. Those eleven categories and their sub categories are defined in the feed report. Figure 3 shows the general migration paths of the NMMSS codes from the nine DOE/DP-22 categories (plus a tenth Section $91 B$ category) to the eleven DOE/MD categories. Table 3 lists the actual NMMSS codes used for each of the eleven main categories and their sub categories.

\footnotetext{
Not an authorized COEI for Plutonium per NMMSS I-17 Report

${ }^{2} \mathrm{COEI}$ is 'Composition of Ending Inventory' as defined by NMMSS I-17 Report

${ }^{3}$ ANSI is American National Standards Institute , Unirradiated Pu Scrap -- Classification, ANSI N15.10 - 1987
} 
To. the right of Table 3 are those COEI and ANSI codes that were not included in the DOE/MD categories because of the lack of significant quantities of those specific materials, and due to the necessity of getting a baseline inventory established quickly. 


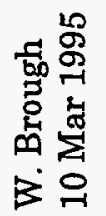

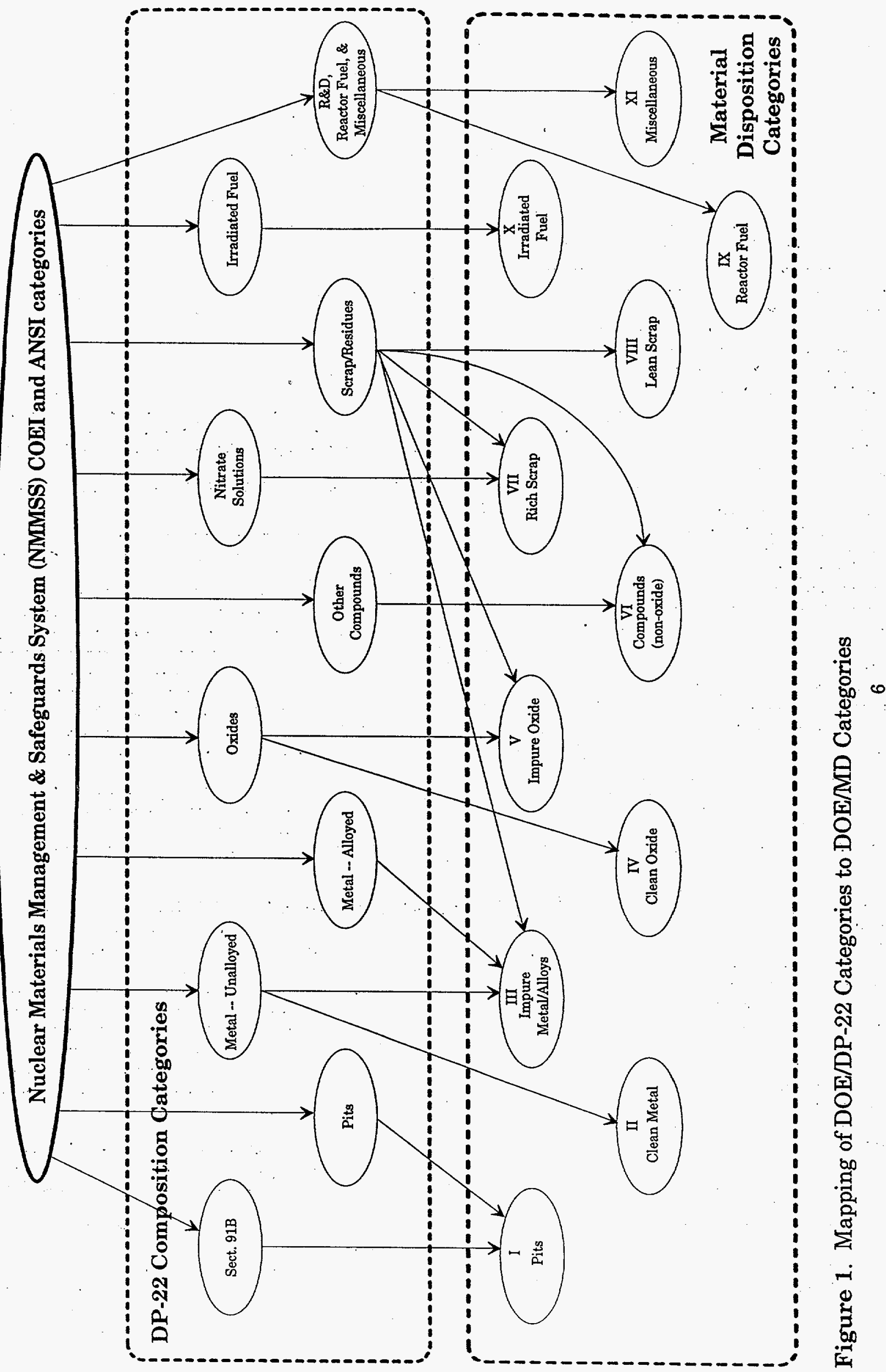


Table 3. Mapping of COEI and ANSI Codes to Material Disposition Categories

\begin{tabular}{|c|c|c|c|c|}
\hline Category & $\begin{array}{c}\text { Sub } \\
\text { Category }\end{array}$ & Description & COEL & Comments \\
\hline
\end{tabular}

L. Pits

L. Pits
\begin{tabular}{|l|c|c|c|c|}
\hline \multicolumn{5}{|c|}{ Sub Categories derived from data external to NMMSS } \\
\hline & & & 309 (-GB-only) \\
\hline & & & 580 & \\
\hline & & & 582 & \\
\hline & & & 772 & \\
\hline & & & 837 & \\
\hline & & & 903 & \\
\hline & & & 905 & \\
\hline & & & 911 & \\
\hline & & & 912 & \\
\hline
\end{tabular}

\section{Clean Metal}

II. Clean Metal
\begin{tabular}{|l|c|l|c|c|}
\hline & 1 & Buttons & 132 & \\
\hline & & & 145 & \\
\hline & 2 & & & \\
\hline & & Billets, Ingots, Castings, \& & 1.50 & \\
\hline & & & 154 & \\
\hline & & & 169 & \\
\hline & & & 195 & \\
\hline & 3 & Weaph Machining & 206 & \\
\hline & & & 224 & \\
\hline & 4 & Recovered Metal, \& Misc. & 690 & \\
\hline & Small Parts & $305(-\mathrm{GB}-$ only) \\
\hline & & & 701 & \\
\hline & & & 779 & \\
\hline & & & 782 & \\
\hline & & & & \\
\hline
\end{tabular}

III. Impure Metal/Alloys

\begin{tabular}{|l|c|l|c|c|}
\hline & 1 & Alloys & 133 & \\
\hline & & & 146 & \\
\hline & & & 151 & \\
\hline & & & 155 & \\
\hline & & & 170 & \\
\hline & & & 196 & \\
\hline & & & 207 & \\
\hline & & & 225 & \\
\hline & & & 257 & \\
\hline & & & 306 (-GB- only) \\
\hline & & & 702 & \\
\hline & & & 722 & B00 \\
\hline & & & 722 & B02 \\
\hline & & & 722 & B03 \\
\hline & & & 722 & B04 \\
\hline & & & 722 & B10 \\
\hline & & & 722 & B13 \\
\hline & & & 722 & B20 \\
\hline & & & 722 & B34 \\
\hline
\end{tabular}

Not yet included:

699

move to Miscellaneous??

move to Miscellaneous??

move to Miscellaneous??

Not-yet included; subs not identified:

134
147
148
152

165
167
180
182
184
197
200
204
220

222
236
237
238

Not yet included; subs not identified:

149

153

166

168

181

183

185

198

201

205

221

223

253

255

256

691 


\begin{tabular}{|c|c|c|c|c|}
\hline & & & 722 & $\mathrm{~B} 40$ \\
\hline & & & 722 & $\mathrm{~B} 41$ \\
\hline & & & 722 & $\mathrm{~B} 50$ \\
\hline & & & 722 & $\mathrm{~B} 70$ \\
\hline & & & 722 & $\mathrm{~B} 80$ \\
\hline & & & 722 & $\mathrm{~B} 90$ \\
\hline & & & 780 & \\
\hline & & & 783 & \\
\hline & 2 & Impure Unalloyed Metal & 721 & $\mathrm{A00}$ \\
\hline & & & 721 & $\mathrm{A01}$ \\
\hline & & & 721 & $\mathrm{A02}$ \\
\hline & & & 721 & $\mathrm{A03}$ \\
\hline & & & 721 & $\mathrm{A04}$ \\
\hline
\end{tabular}

\section{Clean Oxide}

IV. Clean Oxide
\begin{tabular}{|l|l|l|l|l|}
\hline & no sub categories & 453 & \\
\hline & & & 454 & \\
\hline & & & 455 & \\
\hline
\end{tabular}

Not yet included:

060, 061, 062, 235, 287, $289,456,457,635,636$, 637,709

V. Impure Oxide

\begin{tabular}{|c|c|c|c|c|}
\hline . & 1 & Oxides & 697 & \\
\hline & & & 725 & $\mathrm{COO}$ \\
\hline & & & 725 & $\mathrm{C01}$ \\
\hline & & & 725 & $\mathrm{CO} 2$ \\
\hline & & & & \\
\hline & 2 & Pu-DU/NU Oxides & 725 & $\mathrm{C} 40$ \\
\hline & & & 725 & C41 \\
\hline & & & 725 & $\mathrm{C} 43$ \\
\hline & & & 725 & $\mathrm{C50}$ \\
\hline & & & 725 & C51 \\
\hline & & & 725 & $\mathrm{C52}$ \\
\hline & & & & \\
\hline & 3 & Pu-EU Oxides & 725 & C70 \\
\hline & & & 725 & C71 \\
\hline & & & 725 & $\mathrm{C} 72$ \\
\hline & & & 727 & E70 \\
\hline & & 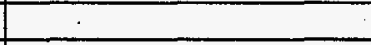 & & \\
\hline & 4 & Pu-Np Oxides & 725 & $\mathrm{C} 90$ \\
\hline & & & & \\
\hline & 5 & Pu-Th Oxides & 725 & $\mathrm{C80}$ \\
\hline & $\therefore$ & $\ldots$ & & $\cdot$ \\
\hline & 6 & Pu-Be Oxides & 725 & $\mathrm{CO3}$ \\
\hline & & & & \\
\hline & 7. & Pu-Zr Oxides & 725 & $\mathrm{CO4}$ \\
\hline
\end{tabular}

\section{Compounds (non-oxide)}

VI. Compounds (non-oxide)
\begin{tabular}{|c|c|l|c|c|}
\hline & 1 & Carbides & 725 & C 25 \\
\hline & & & & \\
\hline & 2 & Hydrides & 725 & C26 \\
\hline & 3 & Nitrides & & \\
\hline & & & 725 & C27 \\
\hline & 4 & Halides & 725 & C 77 \\
\hline & & & & \\
\hline & 5 & Encapsulated Compounds & 725 & C 30 \\
\hline & 6 & & & \\
\hline & & & 776 & \\
\hline
\end{tabular}

This COEI belongs in Rich Scrap, Non-Conforming Scrap??

Not yet included; subs not identified:

063

064

081

082

083

130

131

410

572

692

770

775 
VII. Rich Scrap

\begin{tabular}{|c|c|c|c|c|c|}
\hline \multicolumn{5}{|c|}{ Vil. Rich Scrap } & \multirow{4}{*}{$\begin{array}{l}\text { Includes Lean Scrap } \\
\text { Includes Lean Scrap } \\
\text { Includes Lean Scrap }\end{array}$} \\
\hline & 1 & Graphite & 725 & $\mathrm{C} 75$ & \\
\hline & & & 726 & D04 & \\
\hline & & & 727 & E04 & \\
\hline & & & & & \\
\hline & 2 & Ash / Ash and Soot Heels & 729 & $\mathrm{GO3}$ & Includes Lean Scrap \\
\hline & & & 729 & G73 & Includes Lean Scrap \\
\hline & & & 729 & G93 & Includes Lean Scrap \\
\hline & & & & & \\
\hline & & & & & \\
\hline & 3 & Heels & 729 & G01 & Includes Lean Scrap \\
\hline & & & & & \\
\hline & & & & & $\cdot$ \\
\hline & 4 & $\mathrm{Pu}$ Fluorides & $\frac{725}{725}$ & $\frac{\mathrm{C} 10}{\mathrm{C} 11}$ & \\
\hline & & & 725 & $\mathrm{C} 12$ & \\
\hline & & & & & \\
\hline & & & & & \\
\hline & 5 & $\begin{array}{l}\text { Sand, Slag, \&Crucible } \\
\text { (SS\&C) }\end{array}$ & 729 . & G05 & Includes Lean Scrap \\
\hline & & & & & \\
\hline & & & & & \\
\hline & 6 & Insulation - Filters & 727 & $\mathrm{E} 03$ & Includes Lean Scrap \\
\hline & & & 727 & $\mathrm{E} 05$ & Includes Lean Scrap \\
\hline & 7 & Ceramics & No COEI in & NMMSS & Includes Lean Scrap \\
\hline & & & & & \\
\hline & & & & & \\
\hline & 8 & Sludge & 729 & G02 & Includes Lean Scrap \\
\hline & & & 729 & G40 & Includes Lean Scrap \\
\hline 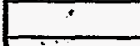 & $\dot{-}$ & & 729 & G70 & Includes Lean Scrap \\
\hline & & & 729 & G90 & Includes Lean Scrap \\
\hline & & & 729 & G92 & Includes Lean Scrap \\
\hline & . & & & & \\
\hline & 9 & $\begin{array}{l}\text { Chloride Salts/ Chloride- } \\
\text { Containing Oxides }\end{array}$ & 725 & $\mathrm{C13}$ & Includes Lean Scrap \\
\hline & & & 729 & G20 & Includes Lean Scrap \\
\hline & & & & & \\
\hline & 10 & Solutions & 409 & & Includes Lean Scrap \\
\hline & & & 700 & & Includes Lean Scrap \\
\hline & & & 703 & & Includes Lean Scrap \\
\hline & & & 728 & FOO & Includes Lean Scrap \\
\hline & & & 728 & F01 & Includes Lean Scrap \\
\hline & & & 728 & $\mathrm{~F} 02$ & Includes Lean Scrap \\
\hline & & & 728 & F03 & Includes Lean Scrap \\
\hline & & & 728 & $\mathrm{~F} 04$ & Includes Lean Scrap \\
\hline & & & 728 & F05 & Includes Lean Scrap \\
\hline & & & 728 & F06 & Includes Lean Scrap \\
\hline . & & & 728 & E07 & Includes Lean Scrap \\
\hline & & & 728 & F40 & Includes.Lean Scrap \\
\hline & & & 728 & F50 & Includes Lean Scrap \\
\hline & & & 728 & F70 & Includes Lean Scrap \\
\hline & & & 728 & F80 & Includes Lean Scrap \\
\hline & & & 728 & $\mathrm{~F} 90$ & Includes Lean Scrap \\
\hline & 11 & Non Conforming Scrap & & & \\
\hline & & & 729 & G00 & Includes Lean Scrap \\
\hline
\end{tabular}

452

693

694

695

696

697

721 (A05)

722 (except B00,

B02, B03, B04,

$\mathrm{B} 10, \mathrm{~B} 11, \mathrm{~B} 13$,

$\mathrm{B} 40, \mathrm{~B} 70, \mathrm{~B} 90)$

(all others except

$\mathrm{COO}, \mathrm{CO}, \mathrm{CO2}$

$\mathrm{C} 25, \mathrm{C} 26, \mathrm{C} 27$,

C30, C41, C71,

C90)

(all others except G04)

VIII. Lean Scrap

\begin{tabular}{l|l|l|l}
1 & Graphite & see Rich Scrap & Reported with Rich Scrap
\end{tabular}




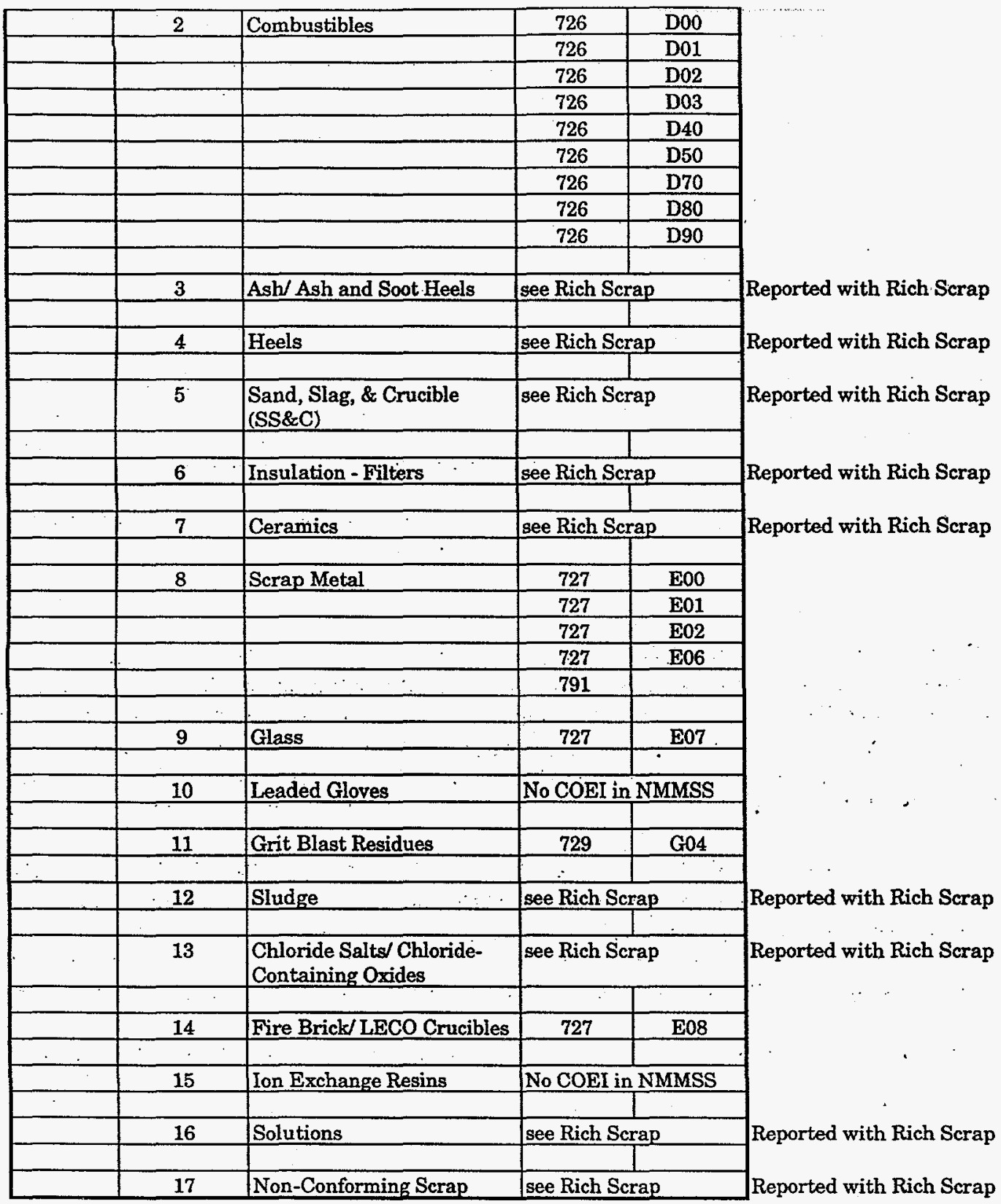

IX. Reactor Fuel

\begin{tabular}{|l|l|l|l|l|}
\hline & & & 285 & \\
\hline & & & 290 & \\
\hline & & & 291 & \\
\hline
\end{tabular}

\section{Irradiated Fuel}

\begin{tabular}{|l|l|l|l|l|}
\hline & & & 360 & \\
\hline & & & 375 & \\
\hline & & & 376 & \\
\hline & & & 385 & \\
\hline & & & 386 & \\
\hline & & & 387 & \\
\hline & & & 390 & \\
\hline & & & 391 & \\
\hline & & & 392 & \\
\hline
\end{tabular}

Not yet included:

362

363

389 


\begin{tabular}{|l|l|l|l|l|}
\hline & & & 405 & \\
\hline & & & 408 & \\
\hline & & & 790 & \\
\hline
\end{tabular}

XI. Miscellaneous

\begin{tabular}{|l|c|l|c|c|}
\hline & 1 & Pu-Al Alloy & TBD & \\
\hline & & & & \\
\hline & 2 & Pu-U-Mo Alloy & 774 & \\
\hline & & & & \\
\hline & 3 & Mixed Oxide & 306 (non-GB-) \\
\hline & & & 309 (non-GB-) \\
\hline & & & 481 & \\
\hline & & & 745 & \\
\hline & & & 746 & \\
\hline & & & 752 & \\
\hline & & & 771 & \\
\hline & & & 781 & \\
\hline & & & 785 & \\
\hline & & & 788 & \\
\hline & & & 836 & \\
\hline
\end{tabular}

Not yet included; subs not identified:

$$
288
$$

305 (non -GB-)

307 (non -GB-)

747

748

749

789

The Material Disposition Categories were formulated to identify potential processing requirements for disposition activities, which might result in separation, purification, volume reduction, stabilization, or immobilization of the material. Any specific material item in the inventory can have several material attributes that will qualify it for a couple of material categories. An attempt was made to avoid this problem, but because of the need to work with some of the pre-existing categories; it was not entirely possible. This made it necessary to generate a sort order algorithm that would provide consistency in how the material is cataloged.

The sort order algorithm of material is as show in Figure 2. The first major sort identifies materials that have been assembled into Pits. It does not matter whether or not a pit is in an assembled weapon. The second major sort identifies Irradiated Fuel. Some material that received very little radiation, resulting in negligible fission products is identified as Miscellaneous material. The third major sort identifies Reactor Fuel. The sorting algorithm then determines the level of impurities in the remaining materials, and groups it into Lean Scrap, Rich Scrap, or (Oxide, Compound, or Metal/Alloy) if the impurity levels are minimal. Several exceptions to this sort algorithm allows for consideration of some specific materials as shown in Figure 2. 


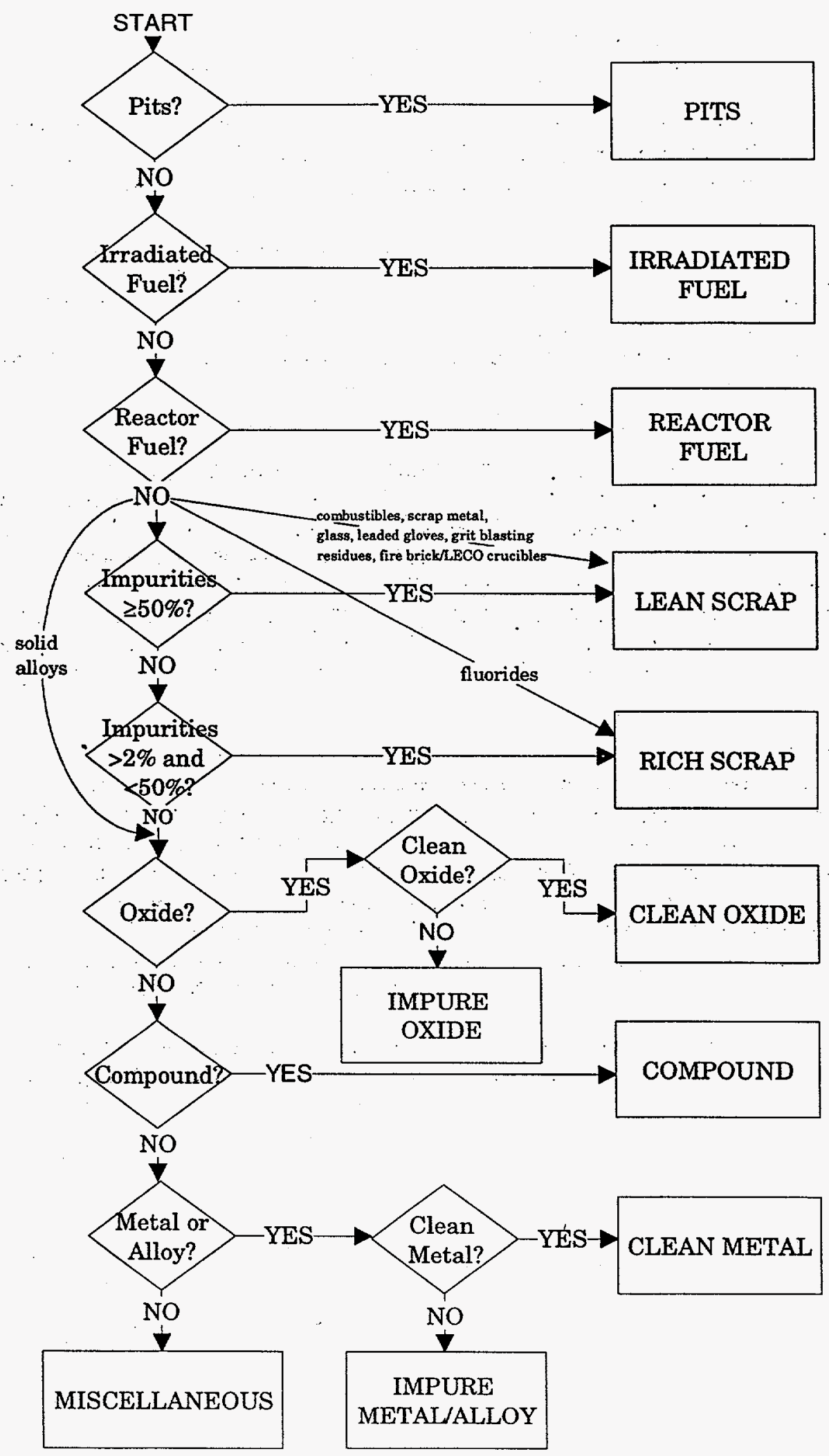

Figure 2. DOE/MD Category Sort Order 


\section{Database Platforms}

The DOE/DP-22 database is installed on an Apple MacIntosh platform using 4th Dimension database software. The DOE/MD database was set up on two separate platforms -- a MacIntosh using 4th Dimension, and a MacIntosh using Microsoft's FoxPro. The purpose of using the FoxPro database platform was to test the capability to import and manipulate the NMMSS data. FoxPro can be run on either an IBM or MacIntosh platform, and both the NAC NMMSS database conversion project and the DOE/AL field office are developing database systems on an IBM platform using FoxPro.

\section{Numerical Calculations}

The material categories were added to the DP-22 (slightly modified NMMSS) database, and then subtotals were derived through queries. The summary tables for the feed report were generated with Microsoft's Excel spreadsheet from data extracted from the database systems. The Pits subcategory totals were generated using the 95-1 P\&PD data entered into an Excel spreadsheet.

The category totals for Rich Scrap and Lean Scap was not as straightforward as were the other categories The proper category for a candidate material sometimes depended on the level of impurities, which is not provided in the NMMSS database. Therefore the candiate materials for Rich Scrap and Lean Scrap were divided into three . categories -- Rich Scrap, Rich+Lean Scrap, and Lean Scrap. The NMMSS database could total those separately. Then information from the Rocky Flats site database was used to ascertain the ratio of Rich-to-Lean in the Rich+Lean category. That ratio was then used to split the Rich+Lean Scrap total into Rich Scrap and Lean Scrap. Those new values were then added to the known quantities of Rich.Scrap and Lean Scrap from the NMMSS totals to derive the final numbers for the Rich Scrap and Lean Scrap categories. That ratio was applied to all the other sites as well. as the actual ratios for the other sites were not available.

\section{Remaining Work}

The work that was performed in support of the feed report provided sufficient information for disposition alternatives to initiate process flow diagrams and assess the requirements for processing the various categories of materials. Onty the NMMSS material categories with significant quantities of reported material were evaluated. Table 3 lists the COEI codes that are used for plutonium, and indicates which of the codes were mapped into the $\mathrm{DOE} / \mathrm{MD}$ categories. The remaining COEI codes should be mapped into the DOE/MD categories so that categories that may later receive significant quantities of material.will show up in the appropriate categories. The same is true for the ANSI scrap codes as shown in Table 3. A complete listing of all ANSI plutonium scrap codes is provided in Table 4, and a complete listing of all material type 50 (Pu-239) COEI entry codes is provided in Table 5. From Tables 4 and 5 it can be seen which codes have not been mapped into the DOE/MD categories:

Additional work will need to be done to make a viable materials database system for the Materials Disposition program. A discussion of the issues and recommendations for a multi-year effort have been forwarded as an R\&D proposal to the DOE/MD. 
Table 4. Mapping of ANSI Scrap Codes to DOE/MD Categories -- Sorted by ANSI.

\begin{tabular}{|c|c|c|c|c|c|c|}
\hline $\begin{array}{l}\text { MD Main } \\
\text { Category } \\
\text { Number }\end{array}$ & $\begin{array}{c}\text { Materials Disposition } \\
\text { Main Category Title }\end{array}$ & $\begin{array}{l}\text { MD Sub } \\
\text { Category } \\
\text { Number }\end{array}$ & MD Sub Category Title & COEI & $\begin{array}{l}\text { ANSI } \\
\text { Scrap } \\
\text { Code }\end{array}$ & ANSI Form \\
\hline III & Impure Metal/Alloys & 2 & Impure Unalloyed Metal & 721 & $\mathrm{~A} 00$ & Unalloyed Metal \\
\hline III & Impure Metal/Alloys & 2 & Impure Unalloyed Metal & 721 & $\mathrm{~A} 01$ & Unalloyed Metal \\
\hline III & Impure Metal/Alloys & 2 & Impure Unalloyed Metal & 721 & $\mathrm{~A} 02$ & Unalloyed Metal \\
\hline III & Impure Metal/Alloys & 2 & Impure Unalloyed Metal & 721 & $\mathrm{~A} 03$ & Unalloyed Metal \\
\hline III & Impure Metal/Alloys & 2 & Impure Unalloyed Metal & 721 & A04 & Unalloyed Metal \\
\hline$?$ & $?$ & $?$ & $?$ & 721 & $\mathrm{~A} 05$ & Unalloyed Metal \\
\hline III & Impure Metal/Alloys & 1 & Alloys & 722 & $\mathrm{~B} 00$ & Alloyed Metal \\
\hline$?$ & $?$ & $?$ & $?$ & 722 & B01 & Alloyed Metal \\
\hline III & Impure Metal/Alloys & 1 & Alloys & 722 & $\mathrm{~B} 02$ & Alloyed Metal \\
\hline III & Impure Metal/Alloys & 1 & Alloys & 722 & B03 & Alloyed Metal \\
\hline III & Impure Metal/Alloys & 1 & Alloys & 722 & B04 & Alloyed Metal \\
\hline$?$ & $?$ & $?$ & $?$ & 722 & B05 & Alloyed Metal \\
\hline III & Impure Metal/Alloys & 1 & Alloys & 722 & $\mathrm{~B} 10$ & Alloyed Metal \\
\hline III & Impure Metal/Alloys & 1 & Alloys & 722 & B11 & Alloyed Metal \\
\hline III & Impure Metal/Alloys & 1 & Alloys & 722 & B13 & Alloyed Metal \\
\hline$?$ & ? & $?$ & $?$ & 722 & B14 & Alloyed Metal \\
\hline III & Impure Metal/Alloys & 1 & Alloys & 722 & $\mathrm{~B} 20$ & Alloyed Metal \\
\hline III & Impure Metal/Alloys & 1 & Alloys & 722 & B34 & Alloyed Metal \\
\hline III & Impure Metal/Alloys & 1 & Alloys & 722 & $\mathrm{~B} 40$ & Alloyed Metal \\
\hline III & Impure Metal/Alloys & 1 & Alloys & 722 & B41 & Alloyed Metal \\
\hline$?$ & $?$ & $?$ & $?$ & 722 & B49 & Alloyed Metal \\
\hline III & Impure Metal/Alloys & 1 & Alloys & 722 & B50 & Alloyed Metal \\
\hline III & Impure Metal/Alloys & 1 & Alloys & 722 & $\mathrm{~B} 70$ & Alloyed Metal \\
\hline III & Impure Metal/Alloys & 1 & Alloys & 722 & $\mathrm{~B} 80$ & Alloyed Metal \\
\hline III & Impure Metal/Alloys & 1 & Alloys & 722 & $\mathrm{~B} 90$ & Alloyed Metal \\
\hline $\mathrm{V}$ & Impure Oxide & 1 & Oxides & 725 & $\mathrm{COO}$ & Compounds \\
\hline V & Impure Oxide & 1 & Oxides & 725 & $\mathrm{C01}$ & Compounds \\
\hline V & Impure Oxide & 1 & Oxides & 725 & $\mathrm{CO} 2$ & Compounds \\
\hline $\mathrm{V}$ & Impure Oxide & 6 & Pu-Be Oxides & 725 & $\mathrm{CO3}$ & Compounds \\
\hline
\end{tabular}




$\begin{array}{cl}\text { V } & \text { Impure Oxide } \\ \text { VII } & \text { Rich Scrap } \\ \text { VII } & \text { Rich Scrap } \\ \text { VII } & \text { Rich Scrap } \\ \text { VII } & \text { Rich Scrap } \\ ? & ? \\ \text { VI } & \text { Compounds (non oxides) } \\ \text { VI } & \text { Compounds (non oxides) } \\ \text { VI } & \text { Compounds (non oxides) } \\ \text { VI } & \text { Compounds (non oxides) } \\ \text { V } & \text { Impure Oxide } \\ \text { V } & \text { Impure Oxide } \\ \text { V } & \text { Impure Oxide } \\ ? & ? \\ ? & ? \\ ? & ? \\ ? & ? \\ \text { V } & \text { Impure Oxide } \\ \text { V } & \text { Impure Oxide } \\ \text { V } & \text { Impure Oxide } \\ \text { VI } & \text { Compounds (non oxides) } \\ ? & ? \\ ? & ? \\ ? & ? \\ \text { V } & \text { Impure Oxide } \\ \text { V } & \text { Impure Oxide } \\ \text { V } & \text { Impure Oxide } \\ ? & ? \\ \text { VII } & \text { Rich Scrap } \\ ? & ? \\ \text { VI } & \text { Compounds (non oxides) } \\ \text { V } & \text { Impure Oxide } \\ ? & ? \\ ? & ? \\ & \end{array}$

Pu-Zr Oxides
Plutonium Fluorides
Plutonium Fluorides
Plutonium Fluorides
Chloride Salts / Chloride-Containing Oxides
$?$
Carbides
Hydrides
Nitrides
Encapsulated Compounds
Pu-DU/NU Oxides
Pu-DU/NU Oxides
Pu-DU/NU Oxides
$?$
$?$
$?$
$?$
Pu-DU/NU Oxides
Pu-DU/NU Oxides
Pu-DU/NU Oxides
Halides
$?$
$?$
$?$
Pu-EU Oxides
Pu-EU Oxides
Pu-EU Oxides
$?$
Graphite
$?$
Nitrides
Pu-Th Oxides
$?$
$?$

\begin{tabular}{lll}
725 & C04 & Compounds \\
725 & C10 & Compounds \\
725 & C11 & Compounds \\
725 & C12 & Compounds \\
725 & C13 & Compounds \\
725 & C14 & Compounds \\
725 & C25 & Compounds \\
725 & C26 & Compounds \\
725 & C27 & Compounds \\
725 & C30 & Compounds \\
725 & C40 & Compounds \\
725 & C41 & Compounds \\
725 & C43 & Compounds \\
725 & C44 & Compounds \\
725 & C45 & Compounds \\
725 & C46 & Compounds \\
725 & C47 & Compounds \\
725 & C50 & Compounds \\
725 & C51 & Compounds \\
725 & C52 & Compounds \\
725 & C54 & Compounds \\
725 & C55 & Compounds \\
725 & C56 & Compounds \\
725 & C57 & Compounds \\
725 & C70 & Compounds \\
725 & C71 & Compounds \\
725 & C72 & Compounds \\
725 & C74 & Compounds \\
725 & C75 & Compounds \\
725 & C76 & Compounds \\
725 & C77 & Compounds \\
725 & C80 & Compounds \\
725 & C81 & Compounds \\
725 & C82 & Compounds \\
& & \\
\hline
\end{tabular}




\begin{tabular}{|c|c|c|c|c|c|c|}
\hline V & Impure Oxide & 4 & Pu-Np Oxides & 725 & C90 & Compounds \\
\hline VIII & Lean Scrap & 2 & Combustibles & 726 & $\mathrm{D} 00$ & Combustibles \\
\hline VIII & Lean Scrap & 2 & Combustibles & 726 & D01 & Combustibles \\
\hline VIII & Lean Scrap & 2 & Combustibles & 726 & $\mathrm{D} 02$ & Combustibles \\
\hline VIII & Lean Scrap & $\overline{2}$ & Combustibles & 726 & $\mathrm{D} 03$ & Combustibles \\
\hline VII & Rich Scrap & 1 & Graphite & 726 & D04 & Combustibles \\
\hline VIII & Lean Scrap & $\overline{2}$ & Combustibles & 726 & $\mathrm{D} 40$ & Combustibles \\
\hline VIII & Lean Scrap & 2 & Combustibles & 726 & D50 & Combustibles \\
\hline VIII & Lean Scrap & 2 & Combustibles & 726 & D70 & Combustibles \\
\hline VIII & Lean Scrap & 2 & Combustibles & 726 & D80 & Combustibles \\
\hline VIII & Lean Scrap & 2 & Combustibles & 726 & D90 & Combustibles \\
\hline VIII & Lean Scrap & 8 & Scrap Metal & 727 & E00 & Noncombustibles \\
\hline VIII & Lean Scrap & 8 & Scrap Metal & $7 \dot{27}$ & E01 & Noncombustibles \\
\hline VIII & Lean Scrap & 8 & Scrap Metal & 727 & E02 & Noncombustibles \\
\hline VII & Rich Scrap & 6 & Insulation - Filters & 727 & E03 & Noncombustibles \\
\hline VII & Rich Scrap & 1 & Graphite & 727 & E04 & Noncombustibles \\
\hline VII & Rich Scrap & 6 & Insulation - Filters & 727 & E05 & Noncombustibles \\
\hline VIII & Lean Scrap & 8 & Scrap Metal & 727 & E06 & Noncombustibles \\
\hline VIII & Lean Scrap & $\dot{9}$ & Glass & 727 & E07 & Noncombustibles \\
\hline VIII & Lean Scrap & 14 & Fire Brick / LECO Crucibles & 727 & E08 & Noncombustibles \\
\hline$?$ & $?$ & $?$ & 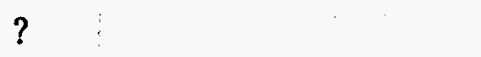 & 727 & E09 & Noncombustibles \\
\hline$?$ & $?$ & $?$ & $?$ & 727 & E40 & Noncombustibles \\
\hline$?$ & $?$ & $?$ & $?$ & 727 & E50 & Noncombustibles \\
\hline V & Impure Oxide & 3 & Pu-EU Oxides & 727 & E70 & Noncombustibles \\
\hline$?$ & $?$ & $?$ & $?$ & 727 & $\mathrm{E} 80$ & Noncombustibles \\
\hline$?$ & $?$ & $?$ & $?$ & 727 & E90 & Noncombustibles \\
\hline VII & Rich Scrap & 10 & Solutions & 728 & F00 & Solutions \\
\hline VII & Rich Scrap & 10 & Solutions & 728 & F01 & Solutions \\
\hline VII & Rich Scrap & 10 & Solutions & 728 & $\mathrm{F02}$ & Solutions \\
\hline VII & Rich Scrap & 10 & Solutions & 728 & F03 & Solutions \\
\hline VII & Rich Scrap & 10 & Solutions & 728 & F04 & Solutions \\
\hline VII & Rich Scrap & 10 & Solutions & 728 & F05 & Solutions \\
\hline VII & Rich Scrap & 10 & Solutions & 728 & F06. & Solutions \\
\hline VII & Rich Scrap & 10 & Solutions & 728 & F07 & Solutions \\
\hline
\end{tabular}




\begin{tabular}{|c|c|c|c|c|c|c|}
\hline VII & Rich Scrap & 10 & Solutions & 728 & F40 & Solutions \\
\hline VII & Rich Scrap & 10 & Solutions & 728 & F50 & Solutions \\
\hline VII & Rich Scrap & 10 & Solutions & 728 & F70 & Solutions \\
\hline VII & Rich Scrap & 10 & Solutions & 728 & F80 & Solutions \\
\hline VII & Rich Scrap & 10 & Solutions & 728 & F90 & Solutions \\
\hline VII & Rich Scrap & 11 & Non-Conforming Scrap & 729 & G00 & Process Residues \\
\hline VII & Rich Scrap & 3 & Heels & 729 & G01 & Process Residues \\
\hline VII & Rich Scrap & 8 & Sludge & 729 & G02 & Process Residues \\
\hline VII & Rich Scrap & 2 & Ash/Ash \& Soot Heels & 729 & G03 & Process Residues \\
\hline VIII & Lean Scrap & 11 & Grit Blasting Residues & 729 & G04 & Process Residues \\
\hline VII & Rich Scrap & 5 & Sand, Slag, and Crucible (SS\&C) & 729 & G05 & Process Residues \\
\hline$?$ & $?$ & $?$ & $?$ & 729 & G10 & Process Residues \\
\hline VII & Rich Scrap & 9 & Chloride Salts / Chloride-Containing Oxides & 729 & G20 & Process Residues \\
\hline VII & Rich Scrap & 8 & Sludge & 729 & G40 & Process Residues \\
\hline$?$ & $?$ & $?$ & $?$ & 729 & G41 & Process Residues \\
\hline$?$ & $?$ & $?$ & $\dot{q}$ & 729 & G42 & Process Residues \\
\hline$?$ & $?$ & $?$ & $?$ & 729 & G43 & Process Residues \\
\hline$?$ & $?$ & $?$ & $?$ & 729 & G50 & Process Residues \\
\hline$?$ & $?$ & $?$ & $?$ & 729 & G51 & Process Residues \\
\hline$?$ & $?$ & $?$ & $?$ & 729 & G52 & Process Residues \\
\hline$?$ & $?$ & $?$ & $?$ & 729 & G53 & Process Residues \\
\hline$?$ & $?$ & $?$ & $?$ & 729 & G57 & Process Residues \\
\hline$?$ & $?$ & $?$ & $?$ & 729 & G58 & Process Residues \\
\hline VII & Rich Scrap & 8 & Sludge & 729 & G70 & Process Residues \\
\hline$?$ & $?$ & $?$ & $?$ & 729 & G71 & Process Residues \\
\hline$?$ & $?$ & $?$ & $?$ & 729 & G72 & Process Residues \\
\hline VII & Rich Scrap & 2 & Ash/Ash \& Soot Heels & 729 & G73 & Process Residues \\
\hline$?$ & $?$ & $?$ & $?$ & 729 & G80 & Process Residues \\
\hline$?$ & $?$ & $?$ & $?$ & 729 & G81 & Process Residues \\
\hline$?$ & $?$ & $?$ & $?$ & 729 & G82 & Process Residues \\
\hline$?$ & $?$ & $?$ & $?$ & 729 & G83 & Process Residues \\
\hline VII & Rich Scrap & 8 & Slụdge & 729 & G90 & Process Residues \\
\hline
\end{tabular}




$\begin{array}{cl}? & ? \\ \text { VII } & \text { Rich Scrap } \\ \text { VII } & \text { Rich Scrap }\end{array}$

$?$ Sludge Ash/Ash \& Soot Heels
729 G91 Process Residues

$729 \quad$ G92 Process Residues

729 G93 Process Residues 
Table 5. Mapping of COEI Codes to DOE/MD Categories -- Sorted by COEI.

\begin{tabular}{|c|c|c|}
\hline $\begin{array}{l}\text { DOE/MD } \\
\text { Category }\end{array}$ & $\begin{array}{l}\text { COEI } \\
\text { Code }\end{array}$ & Composition of Ending Inventory (COED) Description \\
\hline IV.? & 060 & TRIOXIDE FEED \\
\hline IV.? & 061 & DIOXIDE FEED \\
\hline IV.? & 062 & OTHER OXIDE FEED \\
\hline VI.? & 063 & IN HYDROFLUORINATION PROCESS \\
\hline VI.? & 064 & TETRAFLUORIDE PRODUCT \\
\hline VI.? & 081 & TETRAFLUORIDE FEED \\
\hline VI.? & 082 & IN FLUORINATION PROCESS \\
\hline VI.? & 083 & HEXAFLUORIDE PRODUCT \\
\hline VI? & 130 & TETRAFLUORIDE FEED \\
\hline VI.? & 131 & IN REDUCTION PROCESS \\
\hline II.1 & 132 & UNALLOYED BUTTONS PRODUCT \\
\hline III.1 & 133 & ALLOYED BUTTONS PRODUCT \\
\hline II.? & 134 & DERBIES PRODUCT \\
\hline II.I & 145 & UNALLOYED BUTTONS FEED \\
\hline III.1 & 146 & ALLOYED BUTTONS FEED \\
\hline II.? & 147 & DERBIES FEED \\
\hline II.? & 148 & UNALLOYED BRIQUETTES FEED \\
\hline IIII? & 149 & ALLOYED BRIQUETTES FEED \\
\hline II.2 & 150 & UNALLOYED METAL FEED \\
\hline III.1 & 151 & ALLOYED METAL FEED \\
\hline II.? & 152 & IN CASTING PROCESS-UNALLOYED \\
\hline III.? & 153 & IN CASTING PROCESS-ALLOYYED \\
\hline II.2 & 154 & UNALLOYED CASTINGS PRODUCT \\
\hline III.1 & 155 & ALLOYED CASTINGS PRODUCT \\
\hline II.? & 165 & UNALLOYED CASTINGS FEED \\
\hline III.? & 166 & ALLOYED CASTINGS FEED \\
\hline II.? & 167 & IN UNALLOYED ROUGH MACHINING PROCESS \\
\hline III.? & 168 & IN ALLOYED ROUGH MACHINING PROCESS \\
\hline II.2 & 169 & UNALLOYED ROUGH MACHINED ITEMS PRODUCT \\
\hline III.1 & 170 & ALLOYED ROUGH MACHINED ITEMS PRODUCT \\
\hline II.? & 180 & UNALLOYED MACHINE TURNINGS FEED \\
\hline III.? & 181 & ALLOYED MACHINE TUURNINGS FEED \\
\hline II.? & 182 & BRIQUETTING PROCESS-UNALLOYED \\
\hline III.? & 183 & BRIQUETTING PROCESS-ALLOYED \\
\hline II.? & 184 & UNALLOYED BRIQUETTES PRODUCT \\
\hline III? & 185 & ALLOYED BRIQUETTES PRODUCT \\
\hline II.2 & 195 & UNALLOYED INGOTS FEED \\
\hline III.1 & 196 & ALLOYED INGOTS FEED \\
\hline II.? & 197 & UNALLOYED CASTINGS FEED \\
\hline III.? & 198 & ALLOYED CASTINGS FEED \\
\hline II.? & 200 & UNALLOYED ROUGGH MACHINED ITEMS FEED \\
\hline III?? & 201 & ALLOYED ROUGH MACHINED ITEMS FEED \\
\hline II.? & 204 & IN FORMING PROCESS-UNALLLYED \\
\hline III.? & 205 & IN FORMING PROCESS-ALLOYED \\
\hline II. 2 & 206 & UNALLOYED FORMED ITEMS PRODUCT \\
\hline III.1 & 207 & ALLOYED FORMED ITEMS PRODUCT \\
\hline II.? & 220 & UNALLOYED ROUGH MACHINED ITEMS FEED \\
\hline III.? & 221 & ALLOYED ROUGH MACHINED ITEMS FEED \\
\hline II.? & 222 & IN FINISH MACHINING PROCESS-UNALLOYED \\
\hline III.? & 223 & IN FINISH MACHINING PROCESS-ALLOYED \\
\hline II. 3 & 224 & UNALLOYED FINISH MACHINED ITEMS PRODUCT. \\
\hline III.1 & 225 & ALLOYED FINISH MACHINED ITEMS PRODUCT \\
\hline IV.? & 235 & OXIDE FEED \\
\hline II.? & 236 & METAL FEED \\
\hline II? & 237 & IN BILLET FABRICATION PROCESS \\
\hline
\end{tabular}




\begin{tabular}{|c|c|c|}
\hline III? & 238 & BILLETS PRODUCT \\
\hline II.? & 251 & FORMED ITEMS FEED \\
\hline II.? & 252 & UNALLOYED ROUGH MACHINED ITEMS FEED \\
\hline III.? & 253 & ALLOYED ROUGH MACHINED ITEMS FEED \\
\hline II.? & 254 & UNALLOYED FINISH MACHINED ITEMS FEED \\
\hline III? & 255 & ALLOYED FINISH MACHINED ITEMS FEED \\
\hline III.? & 256 & IN CANNING \& CLADDING PROCESS \\
\hline III.1 & 257 & CANNED \& CLAD ITEMS PRODUCT \\
\hline EX.0 & 285 & FORMED ITEMS FEED \\
\hline IV.? & 287 & SPECIAL FUELS OXIDE FEED \\
\hline XI.? & 288 & CANNED \& CLAD ITEMS FEED \\
\hline IV:? & 289 & SINTERED PRODUCTS FEED \\
\hline LXO & 290 & IN FUEL ELEMENT AND TARGET FABR. PROCESS \\
\hline EX.0 & 291 & FABRICATED FUEL ELEMENTS \& TARGETS PRODUCT \\
\hline II.3 & $305(\mathrm{~GB})$ & UNALLOYED FINISH MACHINED ITEMS FEED \\
\hline XI.? & $305(\mathrm{nGB})$ & UNALLOYED FINISH MACHINED ITEMS FEED \\
\hline III.1. & $306(\mathrm{~GB})$ & ALLOYED FINISH MACHINED ITEMS FEED \\
\hline XI.3 & 306(nGB) & ALLOYED FINISH MACHINED ITEMS FEED \\
\hline$? . ?$ & $307(\mathrm{~GB})$ & FABRICATED FUEL ELEMENT, TARGET \& PINS FEED \\
\hline XI.? & 307(nGB) & FABRICATED FUEL ELEMENT, TARGET \& PINS FEED \\
\hline II.? & 308 (GB) & IN ASSEMBLY PROCESS \\
\hline$? ?$ & $308(n G B)$ & IN ASSEMBLY PROCESS \\
\hline 1.0 & $309(\mathrm{~GB})$ & ASSEMBLED ITEMS PRODUCT \\
\hline XI.3 & $309(\mathrm{nGB})$ & ASSEMBLED ITEMS PRODUCT \\
\hline $\mathrm{X} .0$ & 360 & IN REACTOR, FUEL \\
\hline $\mathrm{X} ?$ & 362 & IN REACTOR, TARGETS \\
\hline $\mathrm{X} . ?$ & 363 & IN REACTOR, PRODUCT \\
\hline $\mathrm{X} .0$ & 375 & IRRADIATED RECYCLABLE FUEL \\
\hline $\mathrm{X} .0$ & 376 & IN COOLING, PRODUCT \\
\hline. $\mathrm{X} .0$ & 385 & ALUMINUM PROCESS(IRRADIATED) \\
\hline $\mathrm{X} 0$ & 386 & ZIRCONIUM PROCESS(IRRADIATED) \\
\hline $\mathrm{XO}$ & 387 & ELECTROLYTIC PROCESS(IRRADIATED) \\
\hline $\mathrm{X}$ ? & 389 & U-233 PROCESS(IRRADIATED) \\
\hline X. & 390 & FLUORINEL PROCESS(IRRADIATED) \\
\hline $\mathrm{XO}$ & 391 & CUSTOM OR SPECIAL PROCESS(IRRADIATED) \\
\hline $\mathrm{X} .0$ & 392 & NO PLANNED PROCESS(IRRADIATED) \\
\hline $\mathrm{X} .0$ & 405 & IN COOLING, PRODUCT FEED \\
\hline $\mathrm{X} 0$ & 408 & IN SEPARATION PROCESS \\
\hline VII.10 & 409 & NITRATE SOLUTIONS PRODUCT \\
\hline VI.? & 410 & NITRATE COMPOUNDS PRODUCT \\
\hline II.? & 450 & METAL CHIPS FEED \\
\hline VIr.? & 452 & NITRATE SOLUTIONS FEED \\
\hline IV.0 & 453 & IN OXIDE CONVERSION PROCESS \\
\hline IV.0 & 454 & DIOXIDES PRODUCT \\
\hline IV.0 & 455 & OTHER OXIDES PRODUCT \\
\hline IV.? & 456 & SPECLAL FUELS OXIDE PRODUCT \\
\hline IV.? & 457 & CERAMIC GRADE DIOXIDE PRODUCT \\
\hline$? ?$ & 475 & PROCESS FEED MATERIAL \\
\hline$? ?$ & 480 & IN SOUREE FABRICATION PROCESS \\
\hline XI.3 & 481 & FABRICATED SOURCES PRODUCT \\
\hline VI.? & 572 & HYDRIDES PRODUCT \\
\hline I. 0 & 580 & WEAPONS AWAITING DISASSEMBLY FEED \\
\hline I.0 & 581 & DISASSEMBLY PROCESS \\
\hline 1.0 & 582 & DISASSEMBLED WEAPON COMPONENTS \\
\hline$? ?$ & 583 & INACTIVE STOCKPILE - DOE CUSTODY \\
\hline IV.? & 635 & DIOXIDE POWDER FEED \\
\hline IV.? & 636 & IN SINTERING PROCESS \\
\hline IV.? & 637 & SINTERED PRODUCTS \\
\hline II.4 & 690 & UNALLOYED METAL FEED \\
\hline
\end{tabular}




\begin{tabular}{|c|c|c|}
\hline III.? & 691 & ALLOYED METAL FEED \\
\hline VI.? & 692 & COMPOUNDS FEED \\
\hline VII.? & 693 & OTHER OXIDE FEED \\
\hline VII? & 694 & COMBUSTIBLES FEED \\
\hline VII.? & 695 & NON-COMBUSTIBLES FEED \\
\hline VII.? & 696 & SOLUTIONS FEED \\
\hline V.1 & $697(?)$ & PROCESS RESIDUES \\
\hline VII.? & 697 & PROCESS RESIDUES \\
\hline I.? & 699 & DISASSEMBLED WEAPON COMPONENTS FEED \\
\hline VII.10 & 700 & IN RECOVERY PROCESS-UNIRRADIATED \\
\hline II.4 & 701 & UNALLOYED METAL PRODUCT \\
\hline III.1 & 702 & ALLOYED METAL PRODUCT \\
\hline VII.10 & 703 & NITRATE SOLUTIONS PRODUCT \\
\hline$? . ?$ & 704 & NITRATE COMPOUNDS PRODUCT \\
\hline IV.? & 709 & DIOXIDES PRODUCT \\
\hline $\begin{array}{c}\text { See } \\
\text { ANSI.Table } \\
\end{array}$ & 721 & UNALLOYED METAL(UNIRRADIATED) \\
\hline $\begin{array}{c}\text { See } \\
\text { ANSL.Table } \\
\end{array}$ & 722 & ALLOYED METAL(UNIRRADIATED) \\
\hline $\begin{array}{c}\text { See } \\
\text { ANSI.Table } \\
\end{array}$ & 725 & COMPOUNDS(UNIRRADIATED) \\
\hline $\begin{array}{c}\text { See } \\
\text { ANSI.Table } \\
\end{array}$ & 726 & COMBUSTIBLES(UNIRRADIATED) \\
\hline $\begin{array}{c}\text { See } \\
\text { ANSI.Table }\end{array}$ & 727 & NON-COMBUSTIBLES(UNIRRADLATED) \\
\hline $\begin{array}{c}\text { See } \\
\text { ANSI.Table }\end{array}$ & 728 & SOLUTIONS(UNIRRADIATED) \\
\hline $\begin{array}{c}\text { See } \\
\text { ANSI.Table } \\
\end{array}$ & 729 & PROCESS RESIDUES(UNIRRADIATED) \\
\hline XI.3 & 745 & IN/FOR CRITICAL OR SUB-CRITICAL ASSEMBLIES \\
\hline $\mathrm{XI.3}$ & 746 & IN OTHER R\&D USAGE \\
\hline XI? & 747 & IN/FOR NON-DESTRUCTIVE TESTS \\
\hline XI.? & 748 & IN/FOR EXPERIMENTAL FUEL FABRICATION \\
\hline XI.? & 749 & IN/FOR WEAPONS EFFECT NEU'TRONICS MEASMNTS \\
\hline $\mathrm{XI.3}$ & 752 & IN/FOR WASTE MANAGEMENT R\&D STUDIES \\
\hline VI.? & 770 & CARBIDES \\
\hline $\mathrm{XI.3}$. & 771 & SAMPLES \& STANDARDS \\
\hline $\mathrm{I} .0$ & 772 & FINISHED WEAPONS \\
\hline XI.2 & 774 & EXPERIMENTAL CAPSULES, ELEMENTS, PINS \\
\hline VI.? & 775 & HALOGEN COMPOUNDS NOT REPORTED ELSEWHERE \\
\hline VI.6 & 776 & MISCELLANEOUS COMPOUNDS \\
\hline$? ?$ & 777 & MISCELLANEOUS SOLUTIONS \\
\hline II.? & 778 & BILLETS FOR CRITICALITY STUDIES \\
\hline II.4 & 779 & UNALLOYED ARCHIVE \&/OR RETAINED SAMPLE ITEMS \\
\hline III.I & 780 & ALLOYED ARCHIVE \&/OR RETAINED SAMPLE ITEMS \\
\hline $\mathrm{XI.3}$ & 781 & SEALED SOURCES \\
\hline II.4 & 782 & LABORATORY SAMPLES-UNALLOYED METAL \\
\hline IIII.1 & 783 & LABORATORY SAMPLES-ALLOYED METAL \\
\hline VI.6 & 784 & LABORATORY SAMPLES-COMPOUNDS AND GAS \\
\hline XI.3 & 785 & INVENTORY VERIFICATION SAMPLES \\
\hline XI.3 & 788 & FISSION DETECTORS \\
\hline XI? & 789 & NUCLEAR ACCIDENT DOSIMETERS \\
\hline $\mathrm{X} .0$ & 790 & IRRADIATED MATL FOR FUEL ELEMENT EXAMINATION \\
\hline VIII.8 & 791 & MATERIAL HELD FOR DISPOSITION \\
\hline$? . ?$ & 835 & HELD BY DOE CONTRCTRS(RIS A-Q)NMMSS BK INV \\
\hline XI.3 & 836 & HELD BY DOMESTIC LICENSEES(RIS W-Z)NMMSS BK \\
\hline I.0 & 837 & HELD BY FOREIGN COUNTRIES(RIS R)NMMSS BOOK \\
\hline$? ?$ & 847 & HELD BY DOMESTIC LICENSEES(NMMSS BOOK INV) \\
\hline$? ?$ & 848 & HELD BY FOREIGN COUNTRIES(NMMSS BOOK INV) \\
\hline$? . ?$ & 849 & HELD BY DOE CONTRACTORS(NMMSS BOOK INV) \\
\hline
\end{tabular}




\begin{tabular}{|c|c|c|}
\hline$? ?$ & 860 & IN REACTORS \& CRITICAL ASSEMBLIES \\
\hline$? . ?$ & 861 & IN COOLING \\
\hline$? ?$ & 862 & IN CONVERSION AND FABRICATION PROCESSES \\
\hline$? . ?$ & 863 & IN RECOVERY PROCESSES \\
\hline$? . ?$ & 864 & MATERIAL NOT IN PROCESS \\
\hline$? . ?$ & 865 & UNIRRADIATED SCRAP AWAITING RECOVERY \\
\hline ?.? & 866 & UNIRRADIATED SCRAP AWAITING DISPOSAL \\
\hline$? ?$ & 871 & HELD BY DOMESTIC LICENSEES(NMMSS BOOK INV) \\
\hline ??? & 872 & HELD BY FOREIGN COUNTRIES(NMMSS BOOK INV) \\
\hline$? . ?$ & 874 & HELD BY DOE CONTRACTORS(NMMSS BOOK INV) \\
\hline$? . ?$ & 875 & REPORTED BOOK INVENTORY FOR IAEA \\
\hline $\mathbf{I . 0}$ & 908 & HELD IN WAR RESERVE WEAPONS \\
\hline 1.0 & 905 & RETIRED IN PLACE \\
\hline$? . ?$ & 906 & HELD IN TRAINING CATEGORY \\
\hline$? ?$ & 907 & INACTIVE STOCKPILE - DOD CUSTODY \\
\hline 1.0 & 911 & NÓN-WEAPONS - ARMY \\
\hline 1.0 & 912 & NON-WEAPONS - NAVY \\
\hline$? . ?$ & 913 & NON-WEAPONS - AIR FORCE \\
\hline$? ?$ & 921 & EXPENDITURES \\
\hline$? ?$ & 922 & LOSSES \\
\hline$? . ?$ & 925 & DOD HOLDINGS(NMMSS BOOK INVENTORY) \\
\hline$? ? ?$ & 931 & MILITARY BILATERAL AGREEMENTS (91C) \\
\hline$? ?$ & 932 & MUTUAL DEFENSE PURPOSES (91C) \\
\hline$? ?$ & 933 & LOSSES FOR 91C MATERIAL \\
\hline$? . ?$ & 946 & UNIRRADIATED MATERIAL \\
\hline$? . ?$ & 947 & IRRADIATED MATERLAL \\
\hline$? . ?$ & 956 & AWAITING DISPOSITION-SOLIDS \\
\hline$? ?$ & 957 & AWAITING DISPOSITION-LIQUIDS \\
\hline$? ?$ & 958 & STORED RETRIEVABLY-SOLIDS \\
\hline$? ?$ & 959 & UNDERGROUND WASTE STORAGE-LIQUID \\
\hline$? . ?$ & 960 & UNDERGROUND WASTE STORAGE-CALCINED \\
\hline$? ?$ & 961 & LIQUIDS TO BASINS, PONDS, PITS, OR CRIBS \\
\hline$? . ?$ & 962 & BURIED-SOLIDS $\ldots$ \\
\hline$? ?$ & 963 & BURLAL MATERIAL - (NMMSS BOOK INV) \\
\hline$? . ?$ & 971 & SCRAP GENERATED ON SITE- 6 MONTH PERIOD \\
\hline$? . ?$ & 972 & SCRAP RECOVERED ON SITE- 6 MONTH PERIOD \\
\hline$? ?$ & 973 & SCRAP RECATEGORIZED ON SITE- 6 MONTH PERIOD \\
\hline$? ?$ & 974 & SCRAP DECLARED TO CSMO- 6 MONTH PERIOD \\
\hline$? . ?$ & 991 & COUNTRY OF ORIGIN OR PRODUCTION \\
\hline$? ?$ & 992 & COUNTRY OF ORIGIN OR PROD. (NMMSS BOOK INV) \\
\hline
\end{tabular}

